\title{
miR32-5p promoted vascular smooth muscle cell calcification by upregulating TNFa in the microenvironment
}

\author{
Jingsong Cao ${ }^{1}$, Ling Chen ${ }^{1}$, Xiaoling Zhong ${ }^{1}$, Yingying Shen ${ }^{1}$, Yan Gao ${ }^{1}$, Qian Chen ${ }^{1}$, Xuyu Zu ${ }^{1 *}$ and \\ Jianghua Liu ${ }^{1,2^{*}}$
}

\begin{abstract}
Background: Vascular calcification is often associated with chronic inflammation and is a risk factor for brain arterial stiffness. Our previous results showed that miR32-5p was positively correlated with vascular smooth muscle cells (VSMC) calcification, but it is unclear whether miR32-5p promoted VSMC calcification by regulating inflammatory factor production.

Results: In this study, bioinformatics analysis was used to select tumour necrosis factor a (TNFa) as a candidate inflammatory factor associated with calcification. Moreover, alizarin red staining and qRT-PCR analysis revealed that TNFa produced by BV2 cells was the key promoting factor of VSMC calcification. Interestingly, the expression of TNFa was significantly increased at the mRNA and protein levels after miR32-5p mimic treatment but significantly decreased after miR32-5p antagomir treatment. To explore the mechanism of the regulation of TNFa expression by miR32-5p, bioinformatics analysis indicated that PIKfyve was a candidate target gene of miR32-5p, and luciferase assays verified that the expression of PIKfyve was significantly repressed by miR32-5p mimics. Importantly, rescue experiments showed that the expression of TNFa in BV2 cells treated with miR32-5p antagomir and the PIKfyve inhibitor YM201636 was significantly increased.
\end{abstract}

Conclusions: The production of TNFa in microglia could be affected by miR32-5p targeting PIKfyve, and these results will be beneficial to reveal the mechanism of brain arterial calcification.

Keywords: VSMC calcification, miR32-5p, PIKfyve, TNFa, Microenvironment

\section{Introduction}

Vascular calcification is an independent risk factor for cardio-cerebrovascular diseases [1,2], and its development has been associated with many factors, such as metabolic diseases, vascular diseases and even ageing [3]. Nevertheless, the key link of vascular calcification is a phenotypic change of vascular wall cells, especially the differentiation of vascular smooth muscle cells to osteoid cells [4].

One important characteristic of VSMC calcification is the upregulated expression of calcification-related genes and the downregulated expression of marker genes of

\footnotetext{
*Correspondence: zuxuyu0108@hotmail.com; jianghua990@126.com ${ }^{1}$ Institute of Clinical Medicine, The First Affiliated Hospital of University of South China, Hengyang City 421000, Hunan Province, China

Full list of author information is available at the end of the article
}

smooth muscle cells $[5,6]$. In the calcification process, microRNAs (miRs) play an important role in the regulation of post-transcriptional gene expression [7-9], mediated target mRNA degradation or translational repression $[10,11]$. For example, miR34a promotes VSMC calcification by targeting sirtuin 1 [12], and miR29 contributes to VSMC calcification by mediating elastin downregulation [13]. Interestingly, once VSMC calcification has occurred, calcified VSMCs can produce matrix vesicles or exosomes containing miRs that affect normal cells [14]. Therefore, some researchers have proposed that miRs could be important markers in peripheral blood to predict VSMC calcification [15].

Vascular calcification, similar to other serious diseases, is involved in complicated network mechanisms, and its development is associated with not only changes in miR

(C) The Author(s). 2020 Open Access This article is distributed under the terms of the Creative Commons Attribution 4.0 International License (http://creativecommons.org/licenses/by/4.0/), which permits unrestricted use, distribution, and 
expression but also the induction of inflammation in the microenvironment $[16,17]$. However, the mechanism by which miR affects VSMC calcification by regulating the production of inflammatory factors is still unclear.

Previous research has shown that miR32-5p promotes mouse VSMC calcification by targeting the 3 '-untranslated region of phosphatase and tensin homologue mRNA [18]. In this study, miR transfection, bioinformatics analysis, dot-ELISA, qRT-PCR, luciferase assays and alizarin red staining were used to analyse the influence of miR32-5p on VSMC calcification via the regulation of inflammatory factor production in BV2 cells. The results will be useful to reveal the mechanism of brain arterial calcification.

\section{Materials and methods}

\section{Cell culture}

The mouse microglia cells BV2 and $293 \mathrm{~T}$ cells were purchased from National Infrastructure of Cell Line Resource (China Center for Type Culture Collection), and mouse VSMCs were purchased from Guang Zhou Jennio Biotech Co., Ltd. The cells were cultured in Dulbecco's modified Eagle's medium (DMEM, Gibco BRL, Grand Island, USA) with $10 \%$ foetal calf serum (FBS, Gibco, Australia) and $100 \mathrm{U} / \mathrm{ml}$ penicillin-streptomycin at $37^{\circ} \mathrm{C}$ and $5 \% \mathrm{CO}_{2}$.

\section{Bioinformatics analysis}

The proteins interacting with osteoprotegerin (OPG) were analysed by Cytoscape software. Analysis of the target genes of miR32-5p was performed by using the miRDB, TargetScanVert, and TargetMiner databases and the Gene database of National Center for Biotechnology Information (NCBI). The primers were designed using primer 5 software. The restriction enzyme cutting sites were analysed by DNAMAN software.

\section{miR transfection and YM201636 treatment}

After digestion with trypsin (Beyotime Institute of Biotechnology, China) and centrifugation at $1000 \mathrm{rpm}, \mathrm{BV} 2$ cells were collected and re-suspended in transfection medium. The cells were seeded into 12-well plates and cultured at $37^{\circ} \mathrm{C}$ in $5 \% \mathrm{CO}_{2}$ for $30 \mathrm{~min}$. Following the addition of the premixed solution of Lipofectamine 2000 and miR32-5p mimics, miR32-5p antagomir or negative controls, BV2 cells were cultured at $37^{\circ} \mathrm{C}$ and $5 \% \mathrm{CO}_{2}$ for $6 \mathrm{~h}$. Then, the transfection medium was removed, and the cells were cultured at $37^{\circ} \mathrm{C}$ and $5 \% \mathrm{CO}_{2}$ for $24 \mathrm{~h}$ after the medium was replaced with fresh culture medium or with fresh culture medium containing $0.5 \mu \mathrm{g} / \mathrm{ml}$ LPS (lipopolysaccharide, Beijing Solarbio Science \& Technology Co., Ltd., China).

BV2 cells transfected with miR32-5p antagomir were treated with DMSO (dimethyl sulfoxide) or $10 \mathrm{nM}$
YM201636 (MedChemExpress Co., Ltd., USA), which is a specific inhibitor of PIKfyve (phosphoinositide kinase, FYVE-type zinc finger containing), for $1.5 \mathrm{~h}$, and then the culture medium was replaced with fresh culture medium containing $0.5 \mu \mathrm{g} / \mathrm{ml}$ LPS for $24 \mathrm{~h}$.

\section{Luciferase assay}

The wild-type and mutant sequences of the 3' untranslated region (UTR) of PIKfyve were chemically synthesized, cloned into the pGL3-basic luciferase reporter plasmid (BGI, China) and named PIKfyve-3' UTR Wt (wild type) and PIKfyve-3' UTR Mut (mutation type). Then, 500 ng PIKfyve-3' UTR Wt or PIKfyve-3' UTR Mut and $50 \mathrm{nM}$ miR32 mimics, $100 \mathrm{nM}$ miR-32 antagomir or suitable negative controls (RiboBio Co. Ltd., China) were mixed with Lipofectamine 2000 and then transfected into $293 \mathrm{~T}$ cells. The cells were cultured at $37{ }^{\circ} \mathrm{C}, 5 \% \mathrm{CO}_{2}$ for $48 \mathrm{~h}$. After the cells were harvested, a luciferase assay was carried out with the Dual-Luciferase Reporter Assay System Kit (Promega, San Luis Obispo, CA, USA) at Turner BioSystems (Sunnyvale, CA, USA).

\section{Collection of BV2 cell protein and culture supernatant}

The culture supernatant of BV2 cells was collected after centrifugation at $3000 \mathrm{rpm}$ for $5 \mathrm{~min}$. Following trypsinization and centrifugation at $1000 \mathrm{rpm}$ for $5 \mathrm{~min}$, BV2 cell protein was extracted with RIPA buffer (Beijing Solarbio Science \& Technology Co., Ltd., China). The protein concentration of all samples was detected with a BCA protein concentration detection kit (BOSTER Biological Technology Co. Ltd., China).

\section{Dot-ELISA}

A $1 \mu \mathrm{l}$ sample was dropped into a nitrocellulose (NC) membrane, which had been soaked in PBS (Beijing Solarbio Science \& Technology Co., Ltd., China) for 10 min and dried at room temperature (RT). After the sample was absorbed, the NC membrane was blocked in blocking buffer (PBS containing 5\% nonfat dried milk (BOSTER Biological Technology Co. Ltd., China) and $0.1 \%$ Tween-20) at RT for $1 \mathrm{~h}$ and then washed with $\mathrm{PBS}$ at RT for $5 \mathrm{~min} 3$ times. Subsequently, the NC membrane was incubated with rabbit anti-mouse TNFa (1:300, BOSTER Biological Technology Co. Ltd., China) or rabbit anti-mouse PIKfyve (1:300 Proteintech Group, Inc., USA), which was diluted with blocking buffer at RT for $1 \mathrm{~h}$, and washed with PBS at RT for 5 min 3 times. Following incubation with goat anti-rabbit IgG-HRP (1: 2000, BOSTER Biological Technology Co. Ltd., China) diluted with blocking buffer at RT for $40 \mathrm{~min}$, the NC membrane was washed with PBS at RT for $15 \mathrm{~min} 2$ times. Finally, the protein in the NC membrane was detected by using a Superstar ECL Plus Ready-to-use kit 
(BOSTER Biological Technology Co. Ltd., China) and a FluorChem E system (Cell Biosciences, USA).

\section{Induction of VSMC calcification}

According to the report by Ma et al [19] with some modification, the VSMCs were divided into 6 groups, cultured in 12-well plates with $1 \mathrm{ml}$ DMEM with $10 \% \mathrm{FBS}$ and 100 $\mathrm{U} / \mathrm{ml}$ penicillin-streptomycin, and treated as follows: group 1 received 10\% BV2 culture supernatant (treated with LPS) and $10 \mathrm{mM} \beta$-glycerophosphate (Sigma, Poole, Dorset, UK); group 2 received 10\% BV2 culture supernatant (treated with LPS), which was incubated with $7 \mu \mathrm{l}$ rabbit anti-mouse TNF $\alpha$ antibody (BOSTER Biological Technology Co. Ltd., China) at $4{ }^{\circ} \mathrm{C}$ for $12 \mathrm{~h}$ and $10 \mathrm{mM}$ $\beta$-glycerophosphate; group 3 received $10 \mathrm{mM} \beta$ glycerophosphate and $10 \mathrm{ng} / \mathrm{ml}$ mouse TNF $\alpha$ (BOSTER Biological Technology Co. ltd., China); group 4 received $10 \mathrm{ng} / \mathrm{ml}$ mouse TNF $\alpha$; group 5 received $10 \mathrm{mM} \beta$ glycerophosphate; and group 6 received equal volume of PBS. The VSMCs of all groups were cultured at $37^{\circ} \mathrm{C}$ and $5 \% \mathrm{CO}_{2}$ for 5 days, and the culture supernatants were replaced at day 3 .

\section{RNA extraction and CDNA synthesis}

The BV2 cells in Materials and methods section 2.3 and VSMCs in Materials and methods section 2.7 were respectively collected, total RNA was extracted by RNA simple Total RNA Kit (TianGen Biotech (Beijing) Co., Ltd., China), and cDNA was synthesized by Revert Aid First Strand cDNA Synthesis Kit (Thermo Fisher Scientific Inc., Waltham, MA USA).

\section{qRT-PCR}

The $20 \mu$ reaction volume of qRT-PCR contained $10 \mu \mathrm{l}$ $2 \times$ SYBR Green PCR Mastermix (Beijing Solarbio Science \& Technology Co., Ltd., China), $1 \mu$ l forward primer, and $1 \mu \mathrm{l}$ reverse primer (Table 1), $0.2 \mu \mathrm{l}$ ROX II (Beijing Solarbio Science \& Technology Co., Ltd.,

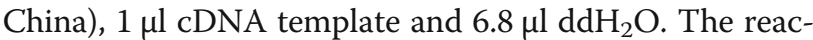
tion programme was as follows: $95^{\circ} \mathrm{C}$ for $5 \mathrm{~min} ; 40 \mathrm{cy}-$ cles at $95^{\circ} \mathrm{C}$ for $15 \mathrm{~s}, 58^{\circ} \mathrm{C}$ for $20 \mathrm{~s}$, and $72{ }^{\circ} \mathrm{C}$ for $30 \mathrm{~s}$. The experiment was performed in ABI 7500 .

\section{Alizarin red staining analysis}

Calcified VSMCs were determined by alizarin red staining. Briefly, after washing with PBS, the VSMCs were fixed with $4 \%$ paraformaldehyde at RT for $15 \mathrm{~min}$. Following staining with Alizarin Red S solution (Beijing Solarbio Science \&Technology Co., Ltd., China) at RT for 2 min, samples were washed with PBS and observed under a light microscope.
Table 1 The primers sequence

\begin{tabular}{ll}
\hline Primer name & Sequence $\left(5^{\prime} \rightarrow 3^{\prime}\right)$ \\
\hline PIKfyve-F & CTGGACTCTGCTAATGATTGC \\
PIKfyve-R & CCTCGCTCTTGTTAAAACGAA \\
TNFa-F & GGCAGGTCTACTTGGAG \\
TNFa-R & TGAGTTACCCGAAAGG \\
Runx2-F & AGTCCCAACTTCCTGTGCT \\
Runx2-R & CTGCTCCGTTCTCAAAGTGG \\
a-SMA-F & GGCATCCACGAAACCACCTAT \\
a-SMA-R & ATGAGACAGACCTAGCCACCG \\
GAPDH-F & TGTTCCTCGTCCCGTA \\
GAPDH-R & TCACCGTTCACCTCTAAC
\end{tabular}

\section{Statistical analysis}

All experiments were repeated at least 3 times. The data were analysed with SigmaPlot 12.0 software and GraphPad Prism 7.00 software, and the results are shown as the mean \pm S.E.M. Student's t test or one-way ANOVA with Bonferroni correction was used to assess statistical significance, and $P<0.05$ or $P<0.01$ were considered significant or very significant, respectively.

\section{Results \\ Exploratory analysis for the inflammatory factor} associated with VSMC calcification

Our previous studies verified that OPG was negatively correlated with VSMC calcification. Thus, Cytoscape software was used to identify OPG interaction proteins. The results showed that the inflammatory factor TNF $\alpha$ (P01375) was a potential regulator involved in VSMC calcification (Fig. 1).

\section{The effect of TNFa expressed by BV2 cells on VSMC calcification}

Furthermore, the effect of TNF $\alpha$ on VSMC calcification was verified (Fig. 2). The results showed that the calcification of VSMCs was promoted by BV2 cell culture supernatant and TNF $\alpha$ after $\beta$-glycerophosphate induction (Fig. 2a, b), whereas BV2 culture supernatant incubated with TNF $\alpha$ antibody (Fig. 2d) or TNF $\alpha$ by itself (Fig. 2e) did not. In addition, qRT-PCR showed that the expression of Runx2 (RUNX family transcription factor 2) significantly increased 2.80 - and 1.34-fold in group 1 and 3 , respectively, and increased 1.08-fold in group 5; the expression of $\alpha$-SMA ( $\alpha$-Smooth muscle actin) decreased 0.95 -fold in group 1 and significantly decreased 0.83 - and 0.43 -fold in group 3 and group 5, respectively (Fig. 2g-i). These results suggested that among the inflammatory factors produced by BV2 cells, TNF $\alpha$ significantly promoted VSMC calcification. 


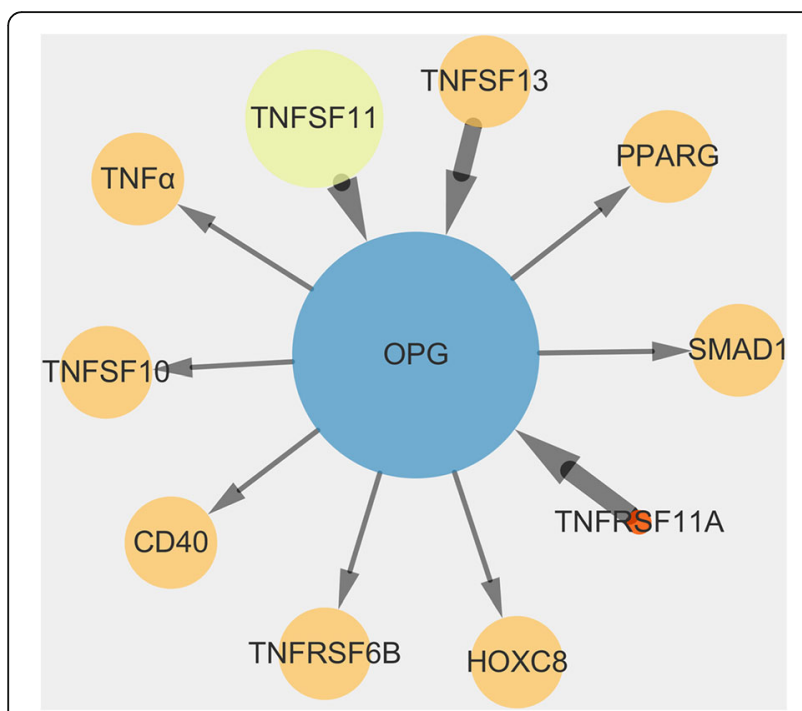

Fig. 1 Search for inflammatory factor associated with VSMC calcification. The protein ID in the circle respectively represented as followed: TNFa, tumor necrosis factor a (P01375); TNFSF13, TNF superfamily member 13 (O75888); TNFSF11, TNF superfamily member 11 (O14788); OPG, TNF receptor superfamily member 11b (O00300); TNFRSF11A, TNF receptor superfamily member 11a (Q9Y6Q6); PPARG, peroxisome proliferator activated receptor gamma (P37231); HOXC8, homeobox C8 (P31273); SMAD1, SMAD family member 1 (Q15797); TNFSF10, TNF superfamily member 10 (P50591); TNFRSF6B, TNF receptor superfamily member 6b (O95407); CD40, CD40 molecule (P25942)

The effect of miR32-5p on TNFa expression in BV2 cells To reveal the roles of miR32-5p in the expression of TNF $\alpha, B V 2$ cells were transfected with miR32-5p mimics or miR32-5p antagomir. The results showed that TNF $\alpha$ mRNA was significantly increased 4.30 -fold after transfection with mimics but significantly decreased 0.63 -fold after antagomir treatment (Fig. 3a). Moreover, a dotELISA assay showed that TNF $\alpha$ in BV2 cells treated with mimics and antagomir significantly increased 1.43-fold and decreased 0.43 -fold, respectively (Fig. 3b, c). The results suggested that miR32-5p promoted the expression of TNF $\alpha$ in BV2 cells.

\section{Identification of the target gene of miR32-5p}

To reveal the mechanism of TNF $\alpha$ expression regulated by miR32-5p, the potential target genes of miR32-5p were searched and analysed by the miRDB, TargetScanVert, TargetMiner and NCBI databases, and PIKfyve was selected as a candidate target gene of miR32-5p (Fig. 4a). Based on the search results in the miRDB database, the sequence of the miR32-5p binding site in PIKfyve (wild type and mutant type) was designed (Fig. 4b, c). Furthermore, luciferase assays verified that the relative luciferase activity in $293 \mathrm{~T}$ cells was significantly downregulated 0.85-fold after cotransfection with PIKfyve 3'UTR-Wt and miR32-5p mimics, while there was no significant change after treatment with PIKfyve 3'UTR-Mut and miR32-5p mimics (Fig. 4d).

In addition, qRT-PCR assay showed that compared with that in the control group, PIKfyve in BV2 cells transfected with miR32-5p mimics or antagomir significantly increased 0.45 -fold or decreased 2.00-fold, respectively (Fig. 4f). Dot-ELISA determined that PIKfyve in BV2 cells transfected with miR32-5p mimics was significantly downregulated 0.81 -fold and significantly upregulated 1.16-fold in BV2 cells transfected with miR325p antagomir (Fig. 4e, g).

\section{PIKfyve inhibited TNFa expression in BV2 cells}

To investigate the effect of PIKfyve on TNF $\alpha$ expression, BV2 cells transfected with miR32-5p antagomir were treated with DMSO or YM201636. After YM201636 treatment, PIKfyve mRNA was significantly decreased 0.82 -fold (Fig. 5a). Moreover, the TNF $\alpha$ mRNA and protein levels increased significantly 1.45- and 4.43-fold, respectively (Fig. $5 \mathrm{~b}-\mathrm{d}$ ). The results suggested that PIKfyve inhibited TNF $\alpha$ production.

\section{Discussion}

Vascular calcification is a complex pathological process. Its development is often involved in diseases with chronic inflammatory disorders [20-22], such as type 2 diabetes mellitus and brain arterial stiffness [2, 23]. In the central nervous system, microglia, which are the largest population of mononuclear phagocytes [24], play important roles in perivascular and pericalcification areas [25]. Interestingly, microglia activation can be induced by high levels of glucose [26], and activated microglia are extensively perivascularly distributed [27].

So, based on previous results that OPG was an inhibitor in VSMC calcification while miR32-5p was a promoter [18], OPG, also named TNF receptor superfamily member $11 \mathrm{~b}$ was selected to explore the inflammatory factors which was associated to VSMC calcification (Fig. 1). Among the candidate proteins, TNF $\alpha$ can promote the development of vascular calcification through interactions with TNF receptor 1 [28-30].

Subsequently, in cytokines produced by microglia, the importance of TNF $\alpha$ for VSMC calcification was analysed. The results showed that the calcification rate of VSMCs was promoted by TNF $\alpha$ (Fig. 2). Moreover, qRT-PCR verified that Runx2 and $\alpha$-SMA were up- and downregulated, respectively, in obviously calcified VSMCs (Fig. 2). Interestingly, VSMC calcification was not obviously affected by TNF $\alpha$ on its own, unless $\beta$ glycerophosphate was added. Thus, TNF $\alpha$ was a key inducer in BV2 cell culture supernatants.

Furthermore, considering higher miR32 levels in plasma of patients with coronary artery calcification, the influence of miR32-5p for microglia producing TNF $\alpha$ 

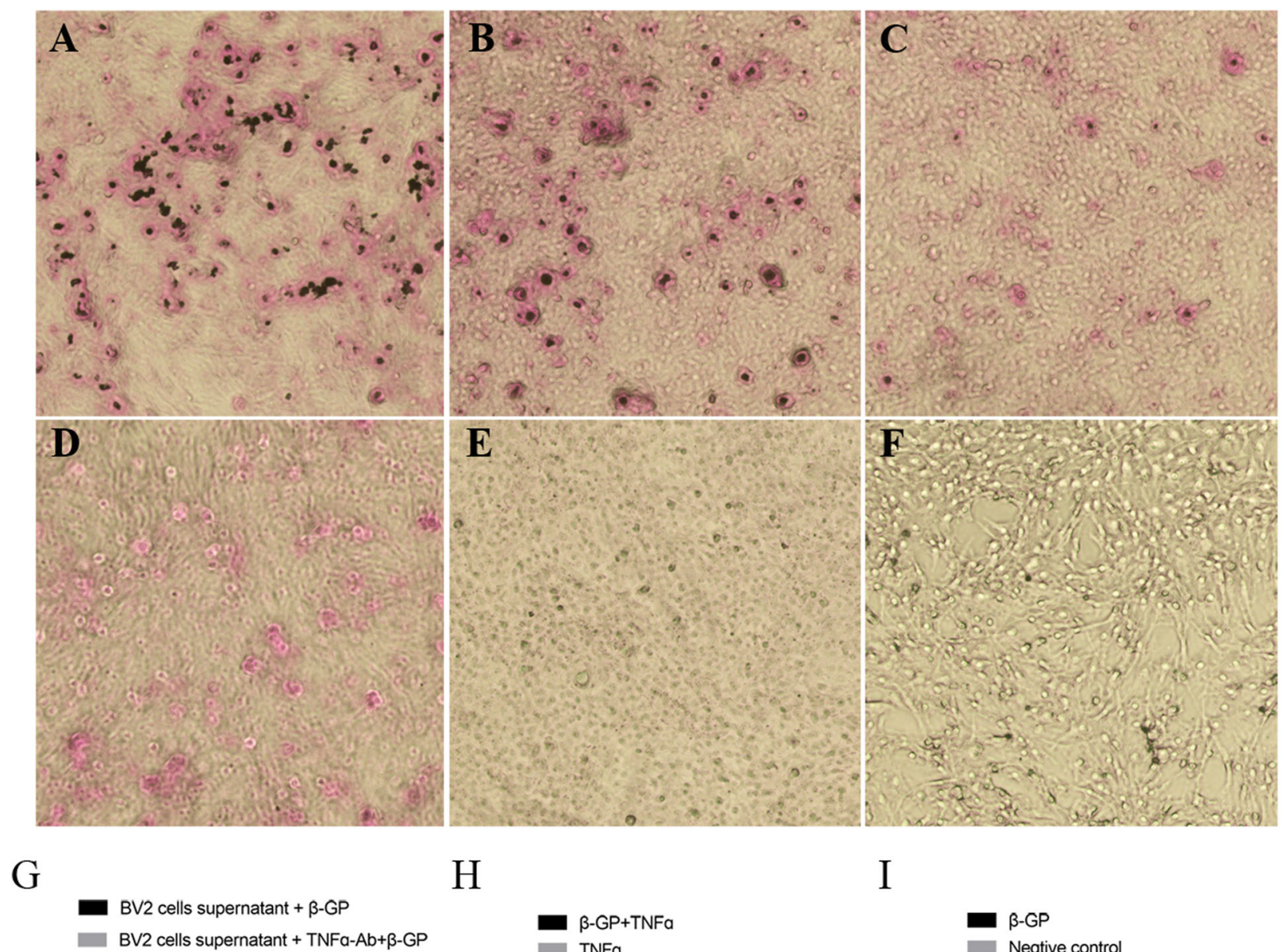

$\mathrm{H}$

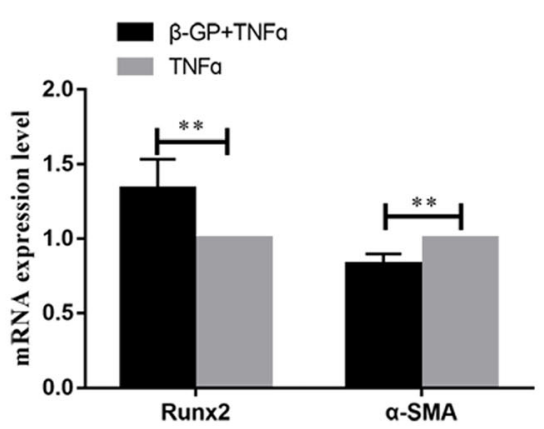

I

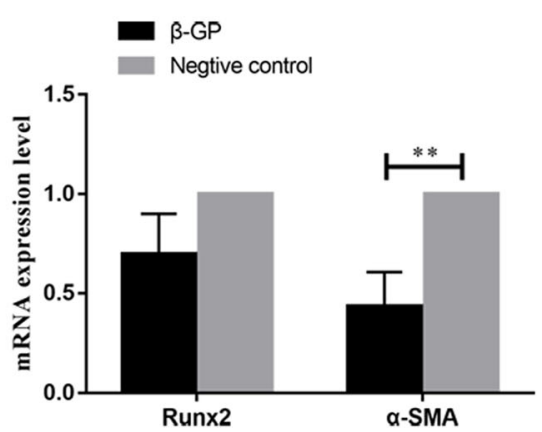

Fig. 2 The influence of TNFa expressed by BV2 cells for VSMC calcification. a-f Alizarin red staining analysis of VSMC calcification in differently stimulated condition (40X), a group1 added 10\% BV2 culture supernatant and $10 \mathrm{mM} \beta$-glycerophosphate, b group3 added $10 \mathrm{mM} \beta$ glycerophosphate and $10 \mathrm{ng} / \mathrm{ml}$ mouse TNFa, c group5 $10 \mathrm{mM} \beta$-glycerophosphate, d group2 added 10\% BV2 culture supernatant, which was incubated with rabbit anti-mouse TNFa antibody, and $10 \mathrm{mM} \beta$-glycerophosphate, e group4 added $10 \mathrm{ng} / \mathrm{ml}$ mouse TNFa, f group6 added equal volume PBS. $\mathbf{g}$-i qRT-PCR analysis for the expression of Runx2 and a-SMA in VSMC, $\mathbf{g}$ the mRNA expression of Runx2 and a-SMA in groups 1 and $2, \mathbf{h}$ the mRNA expression of Runx2 and a-SMA in groups 3 and $4, \mathbf{i}$ the mRNA expression of Runx2 and a-SMA in groups 5 and 6 . Bars represent mean \pm S.D. $(n \geq 3)$. Significant difference marked with one $(p<0.05)$ or two $(p<0.01)$ asterisks

was analysed (Fig. 3). The results showed that TNF $\alpha$ increased expression after miR32-5p mimics treated while decreased expression after miR32-5p antagomir treated. Though the results appeared the regulation of miR32-5p for TNF $\alpha$, these data disagreed with the role of miRNAs, which is degradation or translational repression of the target mRNA [31]. Thus, there is likely a gene that mediates the action of miR32-5p on TNF.
Therefore, the target genes of miR32-5p were explored by bioinformatics method (Fig. 4a). Among the three common genes (RSBN1, PIKfyve and ZFC3H1), PIKfyve plays important roles in the regulation of pleiotropic cellular functions [32-34], for instance regulating the function of macrophage through interfering phagosome and endosome maturation [35]. Meanwhile, luciferase assays appeared that the luciferase activity decreased significantly 


\section{A \\ B}

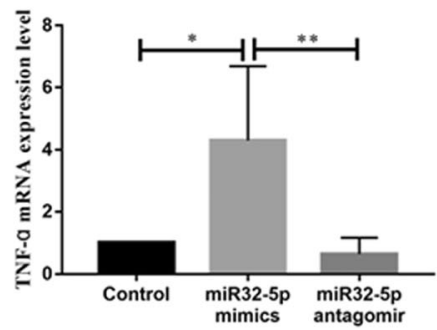

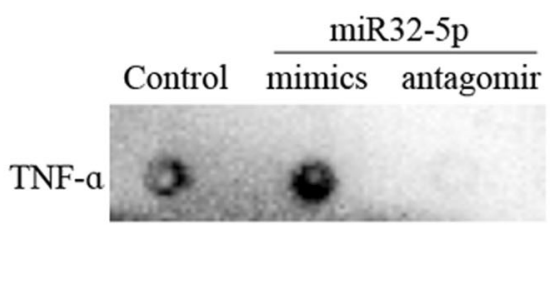

$\mathrm{C}$

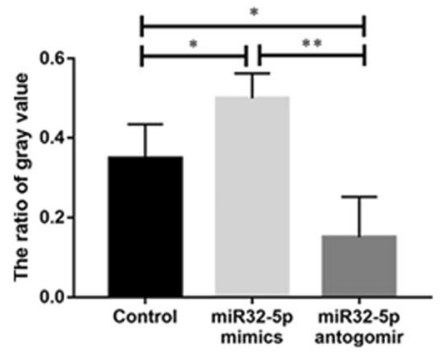

Fig. 3 miR32-5p up-regulated TNFa producing in BV2 cells. a The mRNA expression of TNFa in BV2 cells after LPS stimulated by qRT-PCR analyzed. $\mathbf{b}$ The protein expression of TNFa in BV2 cells after LPS stimulated by Dot-ELISA analyzed. $\mathbf{c}$ The gray value analysis of Dot-ELISA. Bars represent mean \pm S.D. $(n \geq 3)$. Significant difference marked with one $(p<0.05)$ or two $(p<0.01)$ asterisks

A

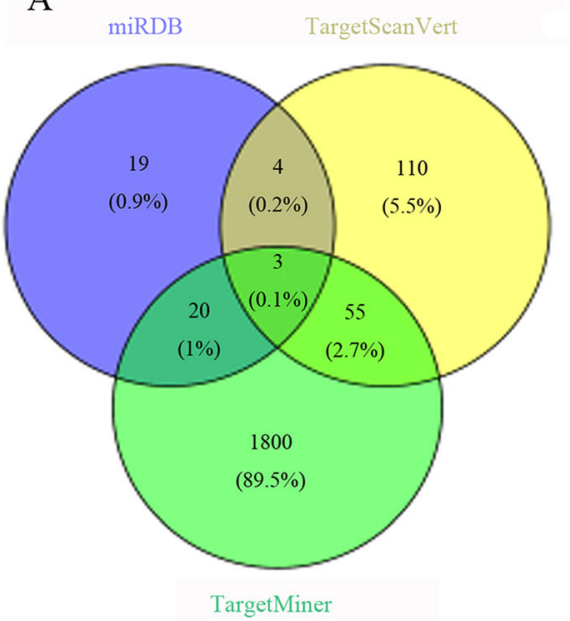

$\mathrm{C}$

Wild type 3' UTR of PIKfyve: 5'...ACUUACGCAGUAUUCAAACCUUCUGUGCAAUGCCUUUUUUUUGGUUGUUUUUGUA... .3' miRNA32-5p:-3' ACGUUGAUUCAUUDCAIIII

Mutant 3' UTR of PIKfyve: 5'...ACUUACGCAGUAUUCAAACCUUCUGCGCAGUGCCUUUUUUUGGUUGUUUUUGUA....3'
B

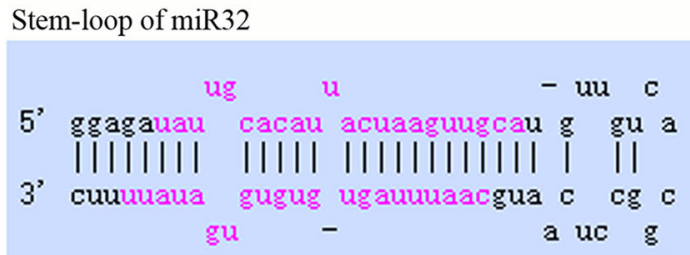

Conserved sites for miR32-5p broadly conserved among vertebrates

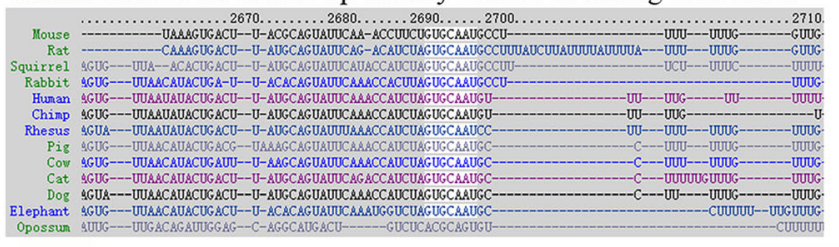

$\mathrm{D}$

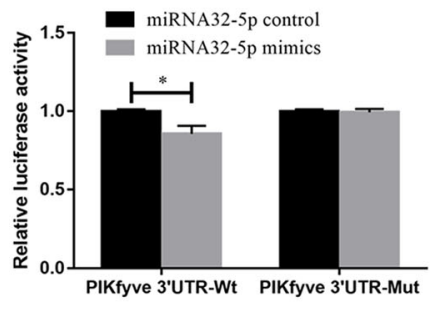

$\mathrm{E}$

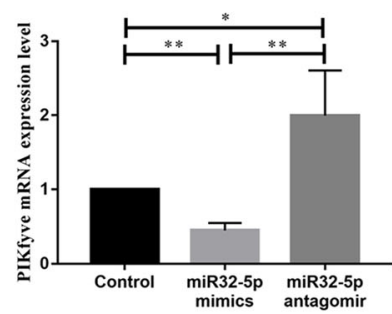

G

$\mathrm{F}$
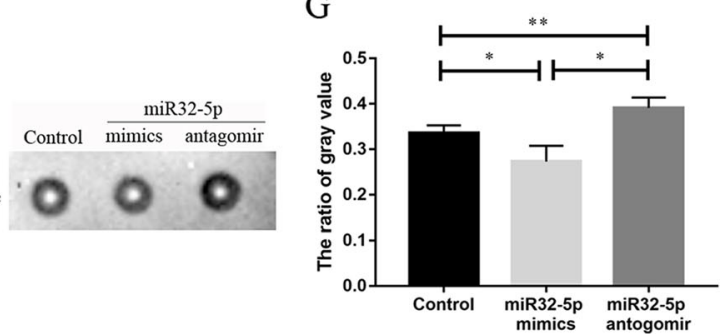

Fig. 4 Analysis of the target gene of miR32-5p. a The candidate target genes of miR32-5p by bioinformatics analyzed, the three genes in intersection were RSBN1, PIKfyve and ZFC3H1. b The stem-loop of miR32 and the conserved sites in the 3' UTR of PIKfyve for miR32-5p. $\mathbf{c}$ The wild type and mutation PIKfyve3' UTR of the seed region of miR-32. d The analysis of miR32-5p target PIKfyve by luciferase assays. e The expression of PIKfyve in BV2 cells after transfected with miR32-5p mimics or antagomir by qRT-PCR analysis. $\mathbf{f}$ The expression of PIKfyve in BV2 cells after transfected with miR32-5p mimics or antagomir by Dot-ELISA analysis. $\mathbf{g}$ The gray value analysis for Dot-ELISA. Bars represent mean \pm S.D. $(n \geq 3)$. Significant difference marked with one $(p<0.05)$ or two $(p<0.01)$ asterisks 

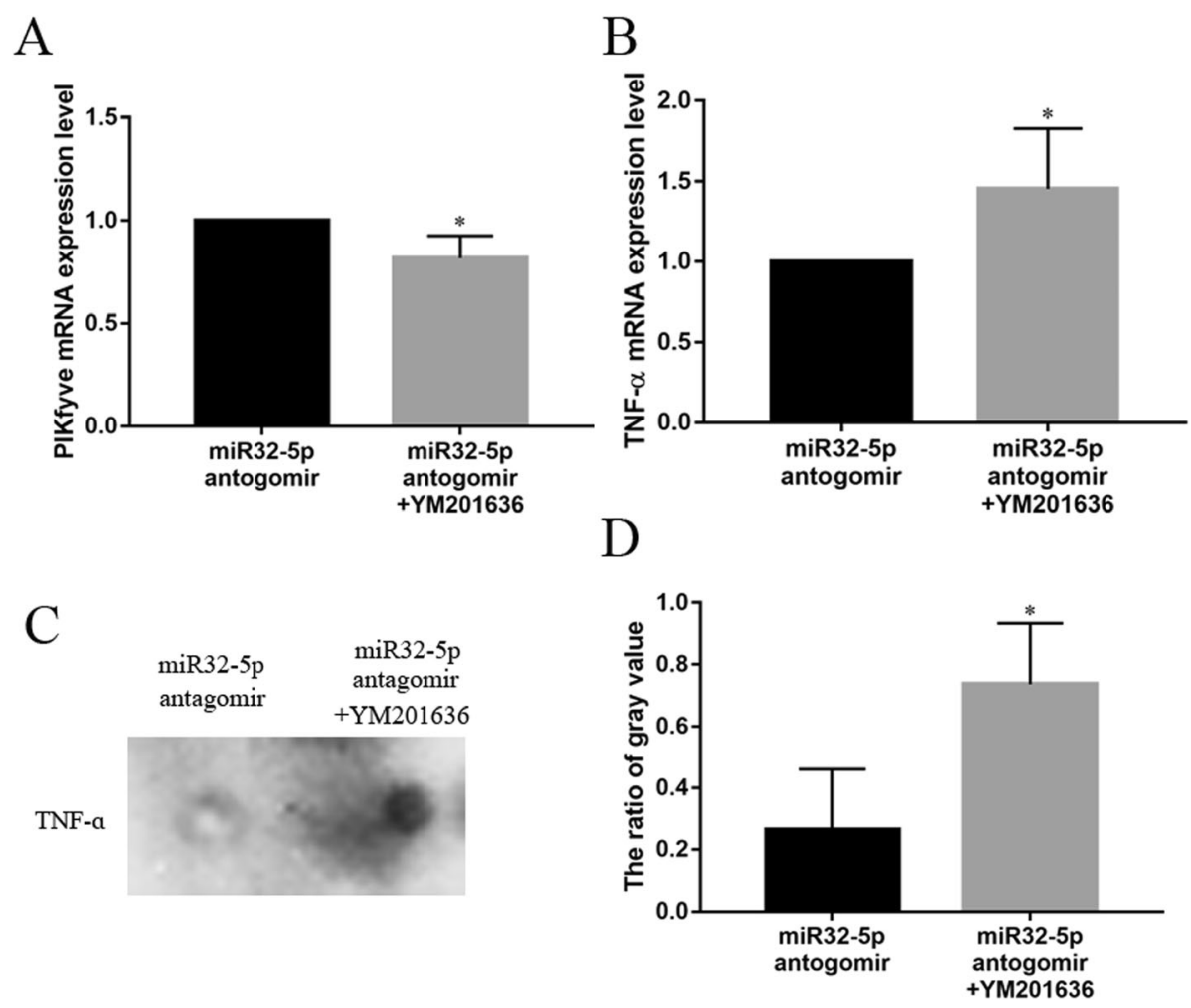

Fig. 5 The influence of PIKfyve for TNFa production in BV2 cells by qRT-PCR and Dot-ELISA analysis. a The expression changes of PIKfyve after treated with YM201636 by qRT-PCR analysis. b The expression changes of TNFa after treated with YM201636 by qRT-PCR analysis. c The expression changes of TNFa after treated with YM201636 by Dot-ELISA analysis. $\mathbf{d}$ The gray value analysis for Dot-ELISA. Bars represent mean \pm S.D. $(n \geq 3)$. Significant difference marked with one $(p<0.05)$ or two $(p<0.01)$ asterisks

after transfecting with miR32-5p mimics and the recombination plasmid of PIKfyve 3'UTR-Wt (Fig. 4b-d). Furthermore, qRT-PCR and dot-ELISA showed that the expression of PIKfyve at the mRNA and protein levels was decreased by miR32-5p expression (Fig. 4f-g). These results suggested that PIKfyve was a target gene of miR32-5p.

To determine the effect of PIKfyve on TNF $\alpha$ production in BV2 cells, a rescue experiment was carried out. After BV2 cells were treated with the miR32-5p antagomir and the PIKfyve-specific inhibitor YM201636, the PIKfyve mRNA was downregulated, while TNF $\alpha$ mRNA was upregulated (Fig. 5a, b). In addition, the production of TNFo in the BV2 cell culture supernatant was increased (Fig. 5c, d). This result was similar to the study by Kawasaki et al., which reported that TNF $\alpha$ production in mouse embryonic fibroblast cells was negatively correlated with the expression of PIKfyve [36]. These results suggested that PIKfyve inhibited the producing of TNF $\alpha$ in BV2 cells.

\section{Conclusions}

Overall, this study found that miR32-5p/PIKfyve/TNF $\alpha$ is a signaling axis that regulates $\mathrm{TNF} \alpha$ production in
BV2 cells, while TNF $\alpha$ could significantly promote VSMC calcification. The results will be valuable to reveal the mechanism of brain arterial calcification.

\section{Abbreviations}

DMEM: Dulbecco's modified Eagle's medium; DMSO: Dimethyl sulfoxide; FBS: Foetal calf serum; LPS: Lipopolysaccharide; a-SMA: a-Smooth muscle actin; miR: microRNA; Mut: Mutation type; NC: Nitrocellulose; NCBI: The Gene database of National Center for Biotechnology Information;

OPG: Osteoprotegerin; PIKfyve: Phosphoinositide kinase, FYVE-type zinc finger containing; RT: Room temperature; Runx2: RUNX family transcription factor 2; TNFa: Tumour necrosis factor a; UTR: Untranslated region; VSMC: Vascular smooth muscle cells; Wt: Wild type

\section{Acknowledgements}

Not applicable.

\section{Authors' contributions}

Laboratory experiments, data analysis and manuscript writing were accomplished by JSC; experimental design and date analysis were accomplished by LC and XLZ; manuscript revise and date analysis were accomplished by YYS; the date analysis was accomplished by YG and QC; the guidance of experimental design and manuscript writing was accomplished by XYZ and JHL. All authors have read and approved the final manuscript.

\section{Funding}

This work was supported by the National Natural Science Foundation of China (No.81170807, No.81873651 and No.81972487), the Major Scientific Research Projects of Hunan health and family planning commission (A2017011), and the Scientific Research Fund of Education Department of 
Hunan Province (No.16B230). The funding body didn't have any role in the design, collection, analysis, and interpretation of data or in the writing of the manuscript.

\section{Availability of data and materials}

All data in the article can be requested from the corresponding author.

\section{Ethics approval and consent to participate}

Not applicable.

\section{Consent for publication}

Not applicable.

\section{Competing interests}

The authors declare that they have no competing interests.

\section{Author details}

${ }^{1}$ Institute of Clinical Medicine, The First Affiliated Hospital of University of South China, Hengyang City 421000, Hunan Province, China. ${ }^{2}$ Department of Metabolism and Endocrinology, The First Affiliated Hospital of University of South China, Hengyang, China.

Received: 20 July 2019 Accepted: 22 October 2019

Published online: 17 January 2020

\section{References}

1. Leopold JA. MicroRNAs Regulate Vascular Medial Calcification. Cells. 2014; 3(4):963-80.

2. Sadekova N, lulita MF, Vallerand D, Muhire G, Bourmoum M, Claing A, Girouard H. Arterial stiffness induced by carotid calcification leads to cerebral gliosis mediated by oxidative stress. J Hypertens. 2018;36(2):286-98.

3. Kwon DH, Kim YK, Kook H. New aspects of vascular calcification: histone Deacetylases and beyond. J Korean Med Sci. 2017;32(11):1738-48.

4. Fuery MA, Liang L, Kaplan FS, Mohler ER 3rd. Vascular ossification: pathology, mechanisms, and clinical implications. Bone. 2018;109:28-34.

5. Bardeesi ASA, Gao J, Zhang K, Yu S, Wei M, Liu P, Huang H. A novel role of cellular interactions in vascular calcification. J Transl Med. 2017:15(1):95.

6. Leopold JA. Vascular calcification: mechanisms of vascular smooth muscle cell calcification. Trends Cardiovasc Med. 2015:25(4):267-74.

7. Dudink E, Florijn B, Weijs B, Duijs J, Luermans J, Peeters F, Schurgers L, Wildberger J, Schotten U, Bijkerk R, et al. Vascular Calcification and not Arrhythmia in Idiopathic Atrial Fibrillation Associates with Sex Differences in Diabetic Microvascular Injury miRNA Profiles. Microrna. 2019;8(2):127-34.

8. Louvet L, Metzinger L, Buchel J, Steppan S, Massy ZA. Magnesium attenuates phosphate-induced deregulation of a MicroRNA signature and prevents modulation of Smad1 and Osterix during the course of vascular calcification. Biomed Res Int. 2016;2016:7419524.

9. Fakhry M, Skafi N, Fayyad-Kazan M, Kobeissy F, Hamade E, Mebarek S, Habib A, Borghol N, Zeidan A, Magne D, et al. Characterization and assessment of potential microRNAs involved in phosphate-induced aortic calcification. J Cell Physiol. 2018;233(5):4056-67.

10. Pan ZW, Lu YJ, Yang BF. MicroRNAs: a novel class of potential therapeutic targets for cardiovascular diseases. Acta Pharmacol Sin. 2010;31(1):1-9.

11. Vickers KC, Rye KA, Tabet F. MicroRNAs in the onset and development of cardiovascular disease. Clin Sci (London, England : 1979). 2014;126(3): 183-94.

12. Badi I, Mancinelli L, Polizzotto A, Ferri D, Zeni F, Burba I, Milano G, Brambilla F, Saccu C, Bianchi ME, et al. miR-34a promotes vascular smooth muscle cell calcification by Downregulating SIRT1 (Sirtuin 1) and AXL (AXL receptor tyrosine kinase). Arterioscler Thromb Vasc Biol. 2018;38(9):2079-90.

13. Sudo R, Sato F, Azechi T, Wachi H. MiR-29-mediated elastin down-regulation contributes to inorganic phosphorus-induced osteoblastic differentiation in vascular smooth muscle cells. Genes Cells. 2015;20(12):1077-87.

14. Zhang C, Zhang K, Huang F, Feng W, Chen J, Zhang H, Wang J, Luo P, Huang $\mathrm{H}$. Exosomes, the message transporters in vascular calcification. J Cell Mol Med. 2018;22(9):4024-33.

15. Howlett P, Cleal JK, Wu H, Shah N, Horton A, Curzen N, Mahmoudi M. MicroRNA 8059 as a marker for the presence and extent of coronary artery calcification. Open Heart. 2018;5(1):e000678.

16. Viegas CSB, Costa RM, Santos L, Videira PA, Silva Z, Araujo N, Macedo AL, Matos $A P$, Vermeer C, Simes DC. Gla-rich protein function as an anti-inflammatory agent in monocytes/macrophages: implications for calcification-related chronic inflammatory diseases. PLoS One. 2017;12(5):e0177829.

17. Bessueille L, Magne D. Inflammation: a culprit for vascular calcification in atherosclerosis and diabetes. Cell Mol Life Sci. 2015;72(13):2475-89.

18. Liu J, Xiao X, Shen Y, Chen L, Xu C, Zhao H, Wu Y, Zhang Q, Zhong J, Tang Z, et al. MicroRNA-32 promotes calcification in vascular smooth muscle cells: implications as a novel marker for coronary artery calcification. PLoS One. 2017;12(3):e0174138.

19. Ma Y, Shi Q, Xiao K, Wang J, Chen C, Gao LP, Gao C, Dong XP. Stimulations of the culture medium of activated microglia and TNF-alpha on a scrapie infected cell line decrease the cell viability and induce marked necroptosis that also occurs in the brains from the patients of human prion diseases. ACS Chem Neurosci. 2019;10(3):1273-83.

20. Nicoll R, Henein M. Arterial calcification: a new perspective? Int J Cardiol. 2017;228:11-22

21. Jackson $A O$, Regine MA, Subrata C, Long S. Molecular mechanisms and genetic regulation in atherosclerosis. Int J Cardiol Heart Vasculature. 2018;21:36-44.

22. Stabley JN, Towler DA. Arterial calcification in diabetes mellitus: preclinical models and translational implications. Arterioscler Thromb Vasc Biol. 2017; 37(2):205-17.

23. Doherty TM, Fitzpatrick LA, Inoue D, Qiao JH, Fishbein MC, Detrano RC, Shah PK, Rajavashisth TB. Molecular, endocrine, and genetic mechanisms of arterial calcification. Endocr Rev. 2004:25(4):629-72.

24. Voet $S$, Prinz M, van Loo G. Microglia in central nervous system inflammation and multiple sclerosis pathology. Trends Mol Med. 2019;25(2):112-23.

25. Nonoda Y, Saito Y, Itoh M, Nakagawa E, Sugai K, Takahashi A, Otsuki T, Saito Y, Arima K, Mizuguchi M, et al. Activation of microglia/macrophages expressing phosphorylated S6 ribosomal protein in a case of hemimegalencephaly with progressive calcification and atrophy. J Neurol Sci. 2009;287(1-2):52-9.

26. Liu Y, Li M, Zhang Z, Ye Y, Zhou J. Role of microglia-neuron interactions in diabetic encephalopathy. Ageing Res Rev. 2018;42:28-39.

27. Davalos D, Ryu JK, Merlini M, Baeten KM, Le Moan N, Petersen MA, Deerinck TJ, Smirnoff DS, Bedard C, Hakozaki H, et al. Fibrinogen-induced perivascular microglial clustering is required for the development of axonal damage in neuroinflammation. Nat Commun. 2012;3:1227.

28. Peng $L$, Zhang $H$, Zhou $Y$, Li W, Jiang $P$, Zhang $Y$, Song K. TNF-alpha suppression and osteoprotegerin overexpression inhibits wear debrisinduced inflammation and osteoclastogenesis in vitro. Int J Artificial Organs. 2015;38(10):565-71.

29. Aghagolzadeh P, Bachtler M, Bijarnia R, Jackson C, Smith ER, Odermatt A, Radpour R, Pasch A. Calcification of vascular smooth muscle cells is induced by secondary calciprotein particles and enhanced by tumor necrosis factoralpha. Atherosclerosis. 2016;251:404-14.

30. Lin CP, Huang PH, Lai CF, Chen JW, Lin SJ, Chen JS. Simvastatin attenuates oxidative stress, NF-kappaB activation, and artery calcification in LDLR-/mice fed with high fat diet via Down-regulation of tumor necrosis factoralpha and TNF receptor 1. PLoS One. 2015:10(12):e0143686.

31. Nanoudis S, Pikilidou M, Yavropoulou M, Zebekakis P. The role of MicroRNAs in arterial stiffness and arterial calcification. An Update and Review of the Literature. Frontiers Genetics. 2017:8:209.

32. Tronchere $\mathrm{H}$, Cinato $M$, Timotin A, Guitou L, Villedieu C, Thibault H, Baetz D, Payrastre B, Valet P, Parini A, et al. Inhibition of PIKfyve prevents myocardial apoptosis and hypertrophy through activation of SIRT3 in obese mice. EMBO Mol Med. 2017:9(6):770-85.

33. Hayashi K, Sasai M, Iwasaki A. Toll-like receptor 9 trafficking and signaling for type I interferons requires PIKfyve activity. Int Immunol. 2015;27(9):435-45.

34. Terajima M, Kaneko-Kobayashi Y, Nakamura N, Yuri M, Hiramoto M, Naitou M, Hattori K, Yokota H, Mizuhara H, Higashi Y. Inhibition of c-Rel DNA binding is critical for the anti-inflammatory effects of novel PIKfyve inhibitor. Eur J Pharmacol. 2016;780:93-105.

35. Kim GH, Dayam RM, Prashar A, Terebiznik M, Botelho RJ. PIKfyve inhibition interferes with phagosome and endosome maturation in macrophages. Traffic (Copenhagen, Denmark). 2014;15(10):1143-63.

36. Kawasaki T, Takemura N, Standley DM, Akira S, Kawai T. The second messenger phosphatidylinositol-5-phosphate facilitates antiviral innate immune signaling. Cell Host Microbe. 2013;14(2):148-58.

\section{Publisher's Note}

Springer Nature remains neutral with regard to jurisdictional claims in published maps and institutional affiliations. 